the pathogenesis of synovitis in spondyloarthritis and degenerative joint disease.

Original article Benjamin M and McGonagle D (2007) Histopathologic changes at "synovio-entheseal complexes" suggesting a novel mechanism for synovitis in osteoarthritis and spondylarthritis. Arthritis Rheum 56: 3601-3609

\section{ANCAs do not predict disease activity in Wegener's granulomatosis}

Antineutrophil cytoplasmic antibodies (ANCAs) are commonly associated with Wegener's granulomatosis. Although ANCAs can provide a useful tool in diagnosing disease, the value of ANCA levels in predicting relapse was controversial. Now, a prospective multicenter study concludes that ANCA levels are only weakly associated with disease activity and, therefore, should not be used to guide decisions relating to immunosuppressive therapy.

Levels of ANCAs recognizing the serine protease proteinase 3 (PR3) and its proenzyme (pro-PR3) were monitored in 156 patients at eight centers across the US by capture enzyme-linked immunosorbent assay. Pro-PR3 ANCAs had previously demonstrated a stronger association with disease activity than ANCAs against PR3; however, no advantage was observed in this study. Overall, the association of ANCA levels and disease severity was weak, consistent with previous reports that ANCA levels are not comparable among patients. A total of 116 patients achieved sustained remission, but the time to sustained remission was not significantly associated with a decrease in ANCA levels. Similarly, increases in PR3-ANCA and pro-PR3-ANCA levels were not associated with relapse: only $40 \%$ of patients who demonstrated an increase in PR3-ANCA level suffered disease relapse within 1 year.

This study has important clinical implications, challenging previous proposals that increased ANCA levels could be used as an indicator for pre-emptive treatment. Given the substantial toxicity associated with the immunosuppressive regimens used to treat Wegener's granulomatosis, better predictors of disease relapse are required to improve long-term therapy.

Original article Finkielman JD et al (2007) Antiproteinase 3 antineutrophil cytoplasmic antibodies and disease activity in Wegener granulomatosis. Ann Intern Med 147: 611-619

\section{Salivary protein and RNA biomarkers for Sjögren's syndrome}

Sjögren's syndrome (SS) can be difficult to diagnose because of the nonspecific symptoms of the disease. Consequently, considerable time can elapse between disease onset and diagnosis. Hu et al., therefore, examined the saliva of patients with primary SS to identify protein and messenger RNA biomarkers that could be used for the early detection of SS.

The study population comprised 10 patients with primary SS and 10 healthy controls matched for age, sex and ethnicity. Patients had significantly lower salivary flow rates than controls, and patient saliva had higher concentrations of total protein and RNA. Ten peptides were found at significantly higher concentrations in patient saliva than in that of controls, and six peptides were found at significantly lower concentrations. In addition, 16 salivary proteins were found downregulated and 25 proteins were found upregulated in primary SS patients compared to matched control subjects. Gene expression analysis showed that mRNAs for 27 genes were significantly overexpressed in saliva of patients compared with controls; the gene for the interferon-inducible protein G1P2 was upregulated almost 500 -fold in patients with SS. Many of the upregulated genes are part of the interferon pathway, suggesting viral involvement in the pathogenesis of SS.

Further studies are planned to validate candidate genes and proteins for a multimarker prediction model for the diagnosis of primary SS.

Original article Hu S et al. (2007) Salivary proteomic and genomic markers for primary Sjögren's syndrome. Arthritis Rheum 56: 3588-3600

\section{Human $T_{H} 17$ induction - treading the line between inflammation and regulation}

Interleukin (IL)-17 is a proinflammatory cytokine that has been implicated in a number of human autoimmune conditions. In animal models, IL-17-producing $\mathrm{CD} 4^{+}$cells, which have been defined as type $17 \mathrm{~T}$ helper $\left(\mathrm{T}_{\mathrm{H}} 17\right)$ cells, are emerging as key perpetrators of immunemediated pathology. Now, Evans et al. have reported the differentiation conditions for 